\title{
EVALUATION AND SELECTION OF KPI IN PROCUREMENT AND DISTRIBUTION LOGISTICS USING SWARA-OFD APPROACH
}

\author{
Vukašin Pajić ${ }^{1}$, Milan Andrejić ${ }^{2}$, Milorad Kilibarda ${ }^{3}$ \\ 1,2,3 University of Belgrade, Faculty of Transport and Traffic Engineering, Vojvode Stepe 305, 11000 \\ Belgrade, Serbia
}

Received 29 November 2020; accepted 20 January 2021

\begin{abstract}
Efficient procurement is one of the key parameters of a company's competitiveness. Besides procurement, distribution plays a significant role in competitiveness as well since the quality of this process directly affects customers. For these reasons, it is necessary for logistics companies to monitor and measure the performance of their procurement and distribution. One of the tools they can use on this occasion is the KPIs. In this paper, 15 KPIs of procurement logistics and $12 \mathrm{KPIs}$ of distribution logistics were analyzed in order to determine the 5 most important ones for each process. In the assessment of the significance of the KPI, 10 experts in the field of logistics participated. The results of their assessment were then used in the SWARA method to obtain the weights of each of the KPIs. After determining the weights, the QFD method was applied to determine the priorities of the observed and analyzed KPIs. The results of the application of these methods showed that order to delivery time, cost per shipment, average delivery time, revenue per order and percentage of on-time deliveries stand out as the five most important KPIs in procurement logistics. On the other hand, total distribution cost, on-time shipping ratio, flexibility of distribution, timeliness of goods delivery and profitability by item stand out as the five most important KPIs in distribution logistics.
\end{abstract}

Keywords: procurement logistics, distribution logistics, SWARA, QFD, KPI.

\section{Introduction}

Procurement logistics, in addition to distribution, is one of the most important segments of the company, given that the efficiency of procurement depends on the ability to meet customer requirements. With this in mind, it can be said that the competitiveness of a company depends on the efficiency of procurement since the satisfaction of customer requirements directly affects the increase in customer satisfaction. In addition to the procurement itself, the procurement logistics is also in charge of market research, supplier analysis, receipt and processing of bids, selection of suppliers, etc. Based on all previously said, it can be concluded that this segment of logistics is of great importance.

As already mentioned, distribution, in addition to procurement, is one of the basic logistics processes. The distribution process begins with the receipt of the order from the customer and ends with the transport process (i.e. unloading at the destination). This process is especially important for the company since with this process company

${ }^{1}$ Corresponding author: v.pajic@sf.bg.ac.rs 
makes contact with the end user. The quality of this process, in addition to procurement, is a key factor in the competitiveness of a logistics company. As distribution is a process that requires a large number of participants, as well as activities, it is necessary to control and manage all activities.

In order to monitor their performance but also to achieve cost savings and increase business efficiency, companies define, measure and monitor KPIs. The purpose of the KPI application is to enable the measurement of achieved performance within the company but also within the industry sector in order to start the benchmark process. Based on the value of the KPI, companies can find out the efficiency of their business. In this paper, two methods were used to assess the significance of KPIs, SWARA and QFD. SWARA is a method where experts apply their own experiences, knowledge and information in order to determine the weight of each attribute. The main feature of the SWARA method is reflected in the possibility of estimating the experts' opinions about the significance of the attributes in the process of weight determination. The first attribute (criterion) after ranking is considered to be the most significant, while the last one is the least significant. The advantage of this method is reflected in the fact that it is not timeconsuming and is not complicated to apply (Mardani et al., 2017). The QFD method was developed with the aim of meeting customer requirements through the design and improvement of products and services. One of the main drivers of this method is the „voice of the customer", where it seeks to determine what users want from the product/ service, and then define the steps to fulfill it. In addition to the "voice of the customer", another basic element of the QFD method is the House of Quality (HoQ). The HoQ consists of user requirements (WHATs), resources (HOWs) and a matrix.

The paper is organized as follows. After the introduction, a description of the problem is given as well as a review of the literature. In the next section of the paper, the methodology used in this paper is presented, which refers to the SWARA and QFD methods. The following section presents the results of the application of the described methods. The last section provides concluding remarks as well as directions for future research.

\section{Problem Description and Literature Review}

The procurement on the one hand includes purchasing and supply management while on the other hand includes managing inbound and outbound logistics. These activities consume a lot of time so it should be managed in order to achieve all the benefits that efficient procurement provides. Also, the task of procurement is supplier selection which can play a significant role as it can influence the delivery lead times. Besides delivery, influence on cycle time also has the procurement transaction process (Bag et al., 2020). Given that procurement logistics can have an impact on a large number of other activities, it is extremely important to monitor and manage this process. Different authors define different procurement logistics KPIs. In the report (USAID, 2013) the authors defined the following KPIs: product price variance, effective contract utilization, expiration management, supplier performance, procurement cycle time, payment processing time, emergency procurement, procurement cost, staff training, transparent price information and 
transparent tendering. On the other hand, Saad et al. (2016) defined the following KPIs: integrity, the productivity of the system, timeliness, quality, price, management, policies and procedures of supplier selection, e-procurement and ease of procurement.

Distribution in addition to procurement is a very complex process. From the very fact that this process includes a large number of activities and participants, a conclusion can be drawn about the importance of this process for a logistics company. The distribution process consists of order processing, warehousing, order picking, packaging, inventory management and transport (Andrejić and Kilibarda, 2016). Distribution efficiency can be significantly affected by vehicle routing, where proper planning can achieve significant cost and time savings. In addition to the choice of routing strategy, distribution processes can be also influenced by the choice of distribution channels, which stands out as an important strategic decision (Andrejić and Kilibarda, 2015). The right choice of the channel can have the effect of reducing costs as well as increasing customer satisfaction. Due to the complexity of the distribution process, it can be concluded that there are a large number of potential KPIs that can be monitored. Some of them are on-time shipping, perfect order percentage, inbound orders received, distribution cost as a percentage of sales, distribution cost per unit shipped, inventory days of supply, reliability of distribution, the flexibility of distribution, the effectiveness of distribution, delivery lead time, on-time delivery, etc.

The aim of this paper is to determine the most important KPIs in procurement and distribution logistics that logistics companies should monitor. To reach this conclusion, the experts were given 15 procurement KPIs (Chan and Qi, 2003; Soni and Kodali, 2010) which were then ranked by importance, from most significant to the least significant. In this paper, the following procurement KPIs were analyzed (adapted from Bressolles and Lang, 2019):

- Inventory cycle time;

- Inventory carrying costs;

- Order to delivery time;

- Order entry time;

- Percentage of shipments arriving in good condition;

- Enquiry-to-response time;

- Percentage of on-time deliveries;

- Stock-out rate;

- Shrinkage;

- Invoice accuracy;

- Shipping accuracy;

- Cost per shipment;

- Average delivery time;

- Revenue per order;

- Average return rate per supplier.

Inventory cycle time represents the time that elapses from the moment when the product enters the warehouse to the moment when the product is shipped from the warehouse. Inventory carrying costs represent the cost of keeping a product in stock which contains material handling costs, inventory capital costs, storage space costs and risk cost. Order to delivery time represents the time that elapses from the moment of ordering to the moment of product delivery to the user. Order entry time represents the time that elapses from the moment of receiving the order to the moment of its entry, i.e. processing. Percentage of shipments arriving in good condition represents the percentage of deliveries without damage observed in relation to the total number of deliveries. Enquiry-to-response time represents the time that elapses from the moment the 
request is received to the moment it is answered. Percentage of on-time deliveries represents the percentage of deliveries that were realized by the requested delivery date. Stock-out rate is the rate of non-stock (when it is not possible to respond to customer requirements). Shrinkage is a commodity that must be written off for one of a number of reasons (defect of goods, expiration date, damage, etc.). Invoice accuracy is a measure of the accuracy of the invoice, i.e. whether all that was requested was delivered. Shipping accuracy is a measure of delivery accuracy, i.e. whether what was requested has been delivered and whether it is in an adequate (requested) quantity. Cost per shipment represents the financial KPI which is expressed as a monetary unit (m.u.) per shipment. Average delivery time is the average time required for delivery, which is obtained as a quotient of the sum of the time required for the realization of certain numbers of deliveries and the number of realized deliveries. Revenue per order, similar to cost per shipment, is a financial KPI that is also expressed in m.u. Average return rate per supplier represents the average number of returns of goods per supplier, where there are various reasons for return such as inadequate quantity, inadequate product, damage, wrong address, etc.

In order to determine the most important KPIs in distribution logistics, the experts were given $12 \mathrm{KPIs}$ which were then ranked by importance, from most significant to the least significant. In this paper, the following distribution KPIs was analyzed (adapted from Krauth et al., 2005; Acumatica, 2018):

- On-time shipping ratio;

- Total distribution cost;

- Flexibility of distribution;

- Profitability by item;
- Timeliness of goods delivery;

- Inventory Turnover Ratio;

- Number of deliveries;

- Product delivered without transaction errors;

- Quality of delivery documentation per truck;

- Effectiveness of distribution planning schedule;

- Realized routes out of planned routes;

- Average delivery planning time.

The on time shipping ratio represents a calculation of the number of order lines shipped on or before the requested ship date versus the total number of order lines. Total distribution cost is usually defined as the cost incurred to deliver the product from the place of origin to the end user. Flexibility of distribution can be defined as the ability to change distribution processes in an efficient or effective manner to adjust to the requirements of both direct and indirect customers (Yu et al., 2012). Profitability by item can be defined as the amount of profit that a particular product (item) makes in a particular period. Timeliness of goods delivery can be defined as on-time delivery i.e. a total number of units delivered within a set period defined by the customer and the supplier. Inventory turnover ratio is a ratio that measures the number of times inventory is sold or consumed in a given time period. Calculating inventory turnover can help companies make better decisions on pricing, procurement, marketing and manufacturing. Number of deliveries represents the total number of deliveries per day/truck/route. Product delivered without transaction errors can be defined as delivery without errors such as the wrong quantity and wrong address. Quality of delivery documentation per truck relates to documentation which has no errors (in quantity, address, items, etc.). 
Effectiveness of the distribution planning schedule represents the degree to which the distribution planning schedule is effective. Realized routes out of planned routes represent how many routes out of planned were realized. Average delivery planning time represents the average time needed for creating a distribution plan.

The importance of both SWARA and QFD methods has been recognized in the literature, as evidenced by a large number of papers in which either only one of the listed methods or a combination of them has been applied. Thus (Radović and Stević, 2018) applied the SWARA method in their paper in order to determine the most important KPIs in transport. The results showed that the coefficient of time utilization, number of $\mathrm{km}$ per vehicle and number of routes per vehicle stood out as the KPIs with the highest importance (priority). Alimardani et al. (2013) used SWARA in agile supplier selection. Stanujkic et al. (2015) used SWARA for the selection of packaging design. Shukla et al. (2016) used SWARA and PROMETHEE to select the best ERP system and competent in the company. Yazdani et al. (2016) in their paper applied a combination of SWARA and QFD methods for the selection of green suppliers.

\section{Methodology}

In order to determine the priorities of KPIs in procurement and distribution logistics, the QFD method was used in this paper. As the application of this method involves determining the weight of each of the KPIs, in order to obtain the value of the total weight for each of the KPIs, the SWARA method was used. The methodological steps of the application of these methods are presented below.

\subsection{SWARA Method}

The procedure for the determination of weights by SWARA method includes the following steps (Yazdani et al., 2016; Radović and Stević, 2018):

Step 1 - All criteria should be sorted in descending order based on their significance evaluated by experts.

Step 2 - Starting from the second criterion, experts express the relative importance of criterion $j$ in relation to the previous j-1 criterion. This way the comparative importance of average value $\left(S_{j}\right)$ is determined for each criterion.

Step 3 - Determine the coefficient $k_{j}$ as follows:

$$
k_{j}=\left\{\begin{array}{c}
1, j=1 \\
s_{j}+1, j>1
\end{array}\right.
$$

Step 4 - Determine the recalculated weight $q_{j}$ as follows:

$q_{j}=\left\{\begin{array}{cc}1 & , j=1 \\ \frac{q_{j-1}}{k_{j}} & , j>1\end{array}\right.$

Step 5 - Calculate the weight values of criteria as follows:

$w_{j}=\frac{q_{j}}{\sum_{k=1}^{n} q_{k}}$

where $w$ j represents the relative weight value of the criterion $j$. 


\subsection{QFD Method}

The process of implementation of the QFD method includes the following steps (adapted from Yazdani et al., 2016):

Step 1 - Identify the WHATs (in this case KPIs).

Step 2 - Identify the HOWs (in this case planning in different sectors).

Step 3 - Assign priority weights to the WHATs (in this paper, SWARA method was used for this step).

Step 4 - Determine the relationship between WHATs and HOWs using a three point scale: weak relationship (1), moderate relationship (3) and strong relationship (9). These relationships are based on the opinion of experts.

Step 5 - Compute the overall priorities of WHATs and HOWs in the following way: the relative weights of the WHATs are multiplied by the strength of the relationship after which a row and column sums are determined in order to obtain the values of the total weights for WHATs and HOWs.

\section{Results}

In order to determine the priority of KPIs, first, it is necessary to determine the weight of each of the observed KPIs in this paper. The previously mentioned KPIs were first ranked according to the importance by experts, after which the other steps described in the previous section of this paper were moved on. The results of the application of the SWARA method for procurement KPI are presented in Table 1 . As more experts (10 experts) were involved in the evaluation, the geometric mean of the judgments was used in order to obtain a single assessment (Bottani et al., 2018).

Table 1

Results of the SWARA Method for Procurement KPI

\begin{tabular}{|c|c|c|c|c|}
\hline KPI & $\mathbf{S}_{\mathbf{j}}$ & $\mathbf{K}_{\mathbf{i}}=\mathbf{S}_{\mathbf{i}}+\mathbf{1}$ & $\mathbf{Q}_{\mathbf{i}}$ & $\mathbf{W}_{\mathbf{j}}$ \\
\hline Order to Delivery Time & - & 1 & 1 & 0.137 \\
\hline Revenue per Order & 0.05 & 1.05 & 0.952 & 0.130 \\
\hline Percentage of on-time Deliveries & 0.2 & 1.2 & 0.793 & 0.109 \\
\hline Enquiry-to-response Time & 0.14 & 1.14 & 0.695 & 0.095 \\
\hline Cost per Shipment & 0.04 & 1.04 & 0.669 & 0.092 \\
\hline Order Entry Time & 0.23 & 1.23 & 0.544 & 0.075 \\
\hline Average Delivery Time & 0.08 & 1.08 & 0.503 & 0.069 \\
\hline Inventory Cycle Time & 0.11 & 1.11 & 0.453 & 0.062 \\
\hline Inventory Carrying Costs & 0.27 & 1.27 & 0.357 & 0.049 \\
\hline Stock-out Rate & 0.09 & 1.09 & 0.327 & 0.045 \\
\hline $\begin{array}{c}\text { Percentage of Shipments } \\
\text { Arriving in Good Condition }\end{array}$ & 0.36 & 1.36 & 0.241 & 0.033 \\
\hline $\begin{array}{c}\text { Average Return Rate } \\
\text { per Supplier }\end{array}$ & 0.06 & 1.06 & 0.227 & 0.031 \\
\hline Shrinkage & 0.07 & 1.07 & 0.212 & 0.029 \\
\hline Shipping Accuracy & 0.13 & 1.13 & 0.188 & 0.026 \\
\hline Invoice Accuracy & 0.39 & 1.39 & 0.135 & 0.019 \\
\hline$\Sigma$ & & & 7.296 & \\
\hline
\end{tabular}


Based on Table 1, it can be concluded that order to delivery time stood out as the most significant KPI with a weight of 0,137 while invoice accuracy stood out as the least significant with a weight of 0,019 . After obtaining these values, the strengths of the relationships between procurement KPIs and planning within specific sectors were determined, Table 2 . For each KPI, the experts then determined the strength of the relationships between that KPI and planning within specific sectors of the company. Thus when looking at order to delivery time, it can be seen that experts assessed a strong relationship (9) between this KPI and ordering, procurement and transport planning and a medium one (3) with the inventory planning process. A strong relationship between revenue per order and order planning has been assessed as the quantities ordered can have a direct impact on revenue. In addition, the medium strength of the relationship (3) with procurement and inventory planning was estimated (where quantities can again have a decisive influence in determining revenue, given that inventories represent tied-up capital). The medium strength of the relationship (3) was estimated between the percentage of on-time deliveries and procurement and inventory planning, given that the level of inventory (and therefore procurement) directly affects the ability of on-time deliveries as well as meeting customer requirements. Transport planning directly affects on-time deliveries and for that reason, a strong relationship was estimated (9). Enquiry-to-response time depends on the order planning process and for that reason, a strong relationship was assessed (9) but also on procurement planning where if several procurements are consolidated into one there is a direct reduction of this time compared to the situation when a large number of smaller procurements are realized. Cost per shipment directly depends on order planning (the larger the order is, the lower unit costs will be), procurement (the larger the procurement is, the lower unit costs will be) and transport (for larger quantities it is possible to use a larger vehicle thus reducing unit costs) and for these reasons, a strong relationship was estimated (9). Order entry time is primarily related to order planning (where the method of ordering can have a great impact on this time) but also to procurement planning (where the frequency of procurement is important). A strong relationship (9) was assessed between average delivery time and procurement planning (if the procurement sector did not require adequate quantity or type of goods, this indicator will directly increase), transport (if adequate vehicle routing is not performed) and inventory (if there is no required quantity in stock then this time increases significantly). Ordering also has an impact on average delivery time and for this reason the medium strength of the relationship was estimated (3). Inventory cycle time, carrying costs and stock-out directly depend on the order and inventory planning (and for that reason a strong relationship was estimated) as well as on order planning where the medium strength of the relationship was estimated (3). Percentage of shipments arriving in good condition directly depends on the transport planning process and for that reason a strong relationship was estimated (9). Average return rate per supplier directly depends on procurement and inventory planning (and for that reason a strong relationship was estimated) but also on ordering planning (where the medium strength of the relationship was estimated) and transport planning (where the weak 
relationship was estimated). Shrinkage is a consequence of poorly planned ordering, procurement and inventory (and for this reason a strong relationship was estimated) but can also be a consequence of poorly planned transport (where damage can occur during transport) and therefore a weak relationship was estimated. Shipping accuracy directly depends on the process of transport planning (and route selection) and procurement planning (when procurement from a supplier is observed). For this reason, a strong relationship was assessed (9). Order and procurement planning has a direct impact on invoice accuracy, which is why a strong relationship has been assessed (9).

Table 2

QFD Matrix for Procurement

\begin{tabular}{|c|c|c|c|c|c|c|c|}
\hline \multirow[b]{2}{*}{ WHATs } & \multirow{2}{*}{$\begin{array}{c}\text { Weight } \\
\text { by } \\
\text { SWARA }\end{array}$} & \multicolumn{4}{|c|}{ HOWs } & \multirow[b]{2}{*}{$\begin{array}{c}\text { Total } \\
\text { Weight }\end{array}$} & \multirow[b]{2}{*}{ Priority } \\
\hline & & $\begin{array}{c}\text { Order } \\
\text { Planning }\end{array}$ & $\begin{array}{c}\text { Procurement } \\
\text { Planning }\end{array}$ & $\begin{array}{l}\text { Transport } \\
\text { Planning }\end{array}$ & $\begin{array}{l}\text { Inventory } \\
\text { Planning }\end{array}$ & & \\
\hline $\begin{array}{l}\text { Order to Delivery } \\
\text { Time }\end{array}$ & 0.137 & 9 & 9 & 9 & 3 & 4.11 & 1 \\
\hline $\begin{array}{l}\text { Revenue per } \\
\text { Order }\end{array}$ & 0.130 & 9 & 3 & & 3 & 1.95 & 4 \\
\hline $\begin{array}{l}\text { Percentage of on- } \\
\text { time Deliveries }\end{array}$ & 0.109 & & 3 & 9 & 3 & 1.635 & 5 \\
\hline $\begin{array}{l}\text { Enquiry-to- } \\
\text { Response Time }\end{array}$ & 0.095 & 9 & & & & 1.14 & 7 \\
\hline Cost per Shipment & 0.092 & 0 & & 9 & & 2.484 & 2 \\
\hline Order Entry Time & 0.075 & 9 & & & & 0.9 & 10 \\
\hline $\begin{array}{l}\text { Average Delivery } \\
\text { Time }\end{array}$ & 0.069 & 3 & 9 & 9 & 9 & 2.07 & 3 \\
\hline $\begin{array}{l}\text { Inventory Cycle } \\
\text { Time }\end{array}$ & 0.062 & 3 & 9 & & 9 & 1.302 & 6 \\
\hline $\begin{array}{l}\text { Inventory Carrying } \\
\text { Costs }\end{array}$ & 0.049 & 3 & 9 & & 9 & 1.029 & 8 \\
\hline Stock-out Rate & 0.045 & 3 & 105 & & 9 & 0.945 & 9 \\
\hline $\begin{array}{l}\text { Percentage of } \\
\text { Shipments Arriving } \\
\text { in Good Condition }\end{array}$ & 0.033 & & & 9 & & 0.297 & 15 \\
\hline $\begin{array}{l}\text { Average Return rate } \\
\text { per Supplier }\end{array}$ & 0.031 & 3 & ه & 1 & 9 & 0.682 & 12 \\
\hline Shrinkage & 0.029 & 9 & & 1 & 9 & 0.812 & 11 \\
\hline Shipping Accuracy & 0.026 & & 9 & 9 & & 0.468 & 13 \\
\hline Invoice Accuracy & 0.019 & 9 & 0.171 & & & 0.342 & 14 \\
\hline Total Weight & & 5.961 & 6.258 & 4.254 & 3.693 & & \\
\hline Priority & & 2 & 1 & 3 & 4 & & \\
\hline
\end{tabular}


Based on Table 2, it can be concluded that order to delivery time, cost per shipment, average delivery time, revenue per order and percentage of on-time deliveries stand out as the five most important KPIs in procurement logistics. These results can provide a good basis for the companies when it comes to choosing KPIs in procurement logistics. On the other hand, the procurement planning process stood out as the most important, followed by the planning of order, transport and inventory. This confirms that the procurement process is one of the most important processes in logistics and that it is necessary for companies to work on efficient management of this process in order to achieve business efficiency.
Procurement logistics management, in addition to increasing efficiency, also reduces costs and increases flexibility in order to meet customer requirements. The implementation of e-procurement achieves even greater benefits such as improved visibility along the entire chain, cost reduction, global procurement, better operational performance, standardized workflow, simplified processes, improved data accuracy, etc.

The results of the application of the SWARA method for distribution KPI are presented in Table 3. In this case, the geometric mean of the judgments in order to obtain a single assessment was applied too.

\section{Table 3}

Results of the SWARA Method for Distribution KPI

\begin{tabular}{|c|c|c|c|c|}
\hline KPI & $\mathbf{S j}$ & $\mathbf{K} \mathbf{j}=\mathbf{S} \mathbf{j}+\mathbf{1}$ & $\mathbf{Q j}$ & $\mathbf{W j}$ \\
\hline On-time Shipping Ratio & - & 1 & 1 & 0.150 \\
\hline Total Distribution Cost & 0.12 & 1.12 & 0.893 & 0.134 \\
\hline Flexibility of Distribution & 0.06 & 1.06 & 0.842 & 0.127 \\
\hline Profitability by Item & 0.08 & 1.08 & 0.780 & 0.117 \\
\hline Timeliness of Goods Delivery & 0.19 & 1.19 & 0.655 & 0.099 \\
\hline Inventory Turnover Ratio & 0.22 & 1.22 & 0.537 & 0.081 \\
\hline Number of Deliveries & 0.25 & 1.25 & 0.430 & 0.065 \\
\hline $\begin{array}{l}\text { Product Delivered without } \\
\text { Transaction Errors }\end{array}$ & 0.07 & 1.07 & 0.402 & 0.060 \\
\hline $\begin{array}{c}\text { Quality of Delivery } \\
\text { Documentation per Truck/Driver }\end{array}$ & 0.11 & 1.11 & 0.362 & 0.054 \\
\hline $\begin{array}{l}\text { Effectiveness of Distribution } \\
\text { Planning Schedule }\end{array}$ & 0.23 & 1.23 & 0.294 & 0.044 \\
\hline $\begin{array}{c}\text { Realized Routes out of } \\
\text { Planned Routes }\end{array}$ & 0.13 & 1.13 & 0.260 & 0.039 \\
\hline Average Delivery Planning Time & 0.37 & 1.37 & 0.190 & 0.029 \\
\hline$\Sigma$ & & & 6.646 & \\
\hline
\end{tabular}


Based on Table 3, it can be concluded that on-time shipping ratio stood out as the most significant KPI with a weight of 0.150 while average delivery planning time stood out as the least significant with a weight of 0.029 . After obtaining these values, the strengths of the relationships between distribution KPIs and planning within specific sectors were determined, Table 4. In contrast to procurement, in distribution, demand, distribution, transport and inventory planning were observed. For each KPI, the experts then determined the strength of the relationships between that KPI and planning within specific sectors of the company. The on-time shipping ratio directly depends on the planning of distribution and transport, since the correct determination of the delivery schedule, as well as the correct routing of the vehicle, directly affect the on-time shipping ratio. For this reason, a strong relationship was estimated (9). In addition, demand planning also has an impact since proper demand forecasting can affect the timely preparation of goods to be distributed thus affecting an increase in the on-time shipping ratio. Distribution and transport planning has a direct impact on total distribution costs. The success of the planning of these processes depends on the number of deliveries and thus the delivery costs, and for that reason a strong relationship has been assessed (9). The medium relationship (3) was estimated between this KPI and demand planning as well as inventory planning. The number of deliveries depends on the efficiency of demand planning, while the efficiency of inventory planning affects the ability to meet customer requirements (where due to the lack of stock there is a need for additional deliveries which affects the increase in total distribution costs). Distribution flexibility directly depends on distribution planning and inventory planning and for this reason a strong relationship has been assessed (9). Profitability by item is closely related to inventory planning, since the efficiency of planning also determines the level of inventory of certain items (where the profitability of items that spend longer in stock is lower compared to those that are shorter in stock). A medium relationship between this KPI and distribution planning was estimated considering that the costs depend on the choice of vehicle and thus the profitability of the item (transporting a large number of items in a larger vehicle leads to lower unit costs per item). Timeliness of goods delivery mostly depends on distribution planning and transport planning, and for that reason a strong relationship was estimated (9). A medium strong relationship was estimated between this KPI and demand planning (since efficient demand planning can influence better distribution planning). Inventory turnover ratio directly depends on the efficiency of inventory planning where a strong relationship was estimated (9). In addition, this KPI is also affected by demand planning (correct demand forecasting can affect inventory turnover), and for that reason the medium relationship has been estimated (3). The estimated relationship between KPI number of deliveries is the same as for the KPI timeliness of goods delivery since the planning effects are the same in both cases. The percentage of delivered items without errors directly depends on the efficiency of distribution and transport planning and for that reason a strong relationship was estimated. 
Table 4

QFD Matrix for Distribution

\begin{tabular}{|c|c|c|c|c|c|c|c|}
\hline \multirow[b]{2}{*}{ WHATs } & \multirow{2}{*}{$\begin{array}{c}\text { Weight } \\
\text { by } \\
\text { SWARA }\end{array}$} & \multicolumn{4}{|c|}{ HOWs } & \multirow[b]{2}{*}{$\begin{array}{c}\text { Total } \\
\text { Weight }\end{array}$} & \multirow[b]{2}{*}{ Priority } \\
\hline & & $\begin{array}{l}\text { Demand } \\
\text { Planning }\end{array}$ & $\begin{array}{c}\text { Distribution } \\
\text { Planning }\end{array}$ & $\begin{array}{l}\text { Transport } \\
\text { Planning }\end{array}$ & $\begin{array}{c}\text { Inventory } \\
\text { Planning }\end{array}$ & & \\
\hline $\begin{array}{l}\text { On Time Shipping } \\
\text { Ratio }\end{array}$ & 0.150 & 3 & & & & 3.15 & 2 \\
\hline $\begin{array}{l}\text { Total Distribution } \\
\text { Cost }\end{array}$ & 0.134 & 3 & & & 3 & 3.216 & 1 \\
\hline $\begin{array}{l}\text { Flexibility of } \\
\text { Distribution }\end{array}$ & 0.127 & & 9 & 9 & & 2.286 & 3 \\
\hline Profitability by Item & 0.117 & & 3 & & 9 & 1.404 & 5 \\
\hline $\begin{array}{l}\text { Timeliness of } \\
\text { Goods Delivery }\end{array}$ & 0.099 & 3 & & 9 & & 2.079 & 4 \\
\hline $\begin{array}{l}\text { Inventory Turnover } \\
\text { Ratio }\end{array}$ & 0.081 & 3 & & & 9 & 0.972 & 8 \\
\hline $\begin{array}{l}\text { Number of } \\
\text { Deliveries }\end{array}$ & 0.065 & 3 & & & & 1.365 & 6 \\
\hline $\begin{array}{l}\text { Product Delivered } \\
\text { without Transaction } \\
\text { Errors }\end{array}$ & 0.060 & & 9 & 9 & & 1.08 & 7 \\
\hline $\begin{array}{l}\text { Quality of Delivery } \\
\text { Documentation per } \\
\text { Truck }\end{array}$ & 0.054 & & 9 & & & 0.486 & 11 \\
\hline $\begin{array}{l}\text { Effectiveness } \\
\text { of Distribution } \\
\text { Planning Schedule }\end{array}$ & 0.044 & & 9 & 2 & & 0.528 & 10 \\
\hline $\begin{array}{l}\text { Realized Routes out } \\
\text { of Planned Routes }\end{array}$ & 0.039 & & 9 & & & 0.702 & 9 \\
\hline $\begin{array}{l}\text { Average Delivery } \\
\text { Planning Time }\end{array}$ & 0.029 & & 9 & 3 & & 0.348 & 12 \\
\hline Total Weight & & 1.587 & 7.56 & 6.285 & 2.184 & & \\
\hline Priority & & 4 & 1 & 2 & 3 & & \\
\hline
\end{tabular}

Distribution planning has the greatest impact on the quality of delivery documentation per truck (since one of the basic steps in distribution planning is the preparation of documentation that accompanies the goods). The efficiency of distribution planning schedule directly affects the effectiveness of the distribution planning schedule and for that reason a strong relationship has been assessed. This KPI can also be influenced by the efficiency of transport (where due to poor vehicle routing, the effectiveness of the distribution schedule can be affected) and for that reason the medium relationship was estimated. KPI realized routes out of planned routes is closely related and depends on distribution planning (depends on an adequate estimate of the time required for distribution) and transport planning (depends on how the vehicle is routed). In addition to distribution planning itself (where a strong 
relationship was estimated), transport planning can have an impact on average delivery planning time as well (since transport planning is one of the constituent activities during distribution planning).

Based on Table 4, it can be concluded that total distribution cost, on time shipping ratio, flexibility of distribution, timeliness of goods delivery and profitability by item stand out as the five most important KPIs in distribution logistics. These results can provide a good basis for the companies when it comes to choosing KPIs in distribution logistics. On the other hand, the distribution planning process stood out as the most important, followed by the transport planning, inventory planning and demand planning. The results show that besides procurement planning, distribution planning represents one of the most important processes in logistics which needs to be monitored. Effective distribution besides customers can have a significant effect on costs and time savings. For this reason, numerous companies invest in routing software in order to achieve both cost and time savings. One of the significant problems which arise in distribution is last-mile delivery which should be in focus for logistics companies in order to enhance distribution efficiency.

\section{Conclusion}

As procurement logistics and distribution logistics are one of the key factors of a company's competitiveness, it is very important to select and monitor adequate KPIs. In this paper, 15 KPIs of procurement logistics and 12 KPIs of distribution logistics were observed in order to determine 5 key (most important) ones for each process that logistics companies should monitor. In order to determine the 5 most important KPIs, a combination of SWARA and QFD methods was used. The SWARA method was used to determine the weights of each of the KPIs, which is one of the steps of the QFD method. After determining the weights, the HoQ matrix was filled in order to obtain the values of total weights per KPI. The results of this research showed that order to delivery time, cost per shipment, average delivery time, revenue per order and percentage of on-time deliveries stand out as the five most important KPIs in procurement logistics. Total distribution cost, on time shipping ratio, flexibility of distribution, timeliness of goods delivery and profitability by item stand out as the five most important KPIs in distribution logistics. In addition to the KPI priorities, based on the QFD matrix, it was determined that the procurement planning process stood out as the most important when observing procurement and distribution planning stood out as the most important process when observing distribution, which is a confirmation of the importance of this processes. The implementation of the described methodology on the other logistics subsystems in order to determine the most significant KPIs that need to be monitored, stands out as the direction of future research. In addition, the application of other methods for KPI assessment in order to compare the obtained results stands out as a direction of future research as well.

\section{Acknowledgements}

This paper was supported by the Ministry of Education, Science and Technological development of the Republic of Serbia, through the project TR 36006.

\section{References}

Acumatica. 2018. Key Performance Indicators for Distribution. Available from Internet: <https:// www.pcbennett.com/wp-content/uploads/2019/10/ Acumatica-Whitepaper-KPIs-for-Distribution.pdf $>$. 
Alimardani, M.; Hashemkhani Zolfani, S.; Aghdaie, M.H.; Tamošaitiene, J. 2013. A novel hybrid SWARA and VIKOR methodology for supplier selection in an agile environment, Technological and Economic Development of Economy 19: 533-548.

Andrejić, M.; Kilibarda, M. 2015. Distribution channels selection using PCA-DEA approach, International Journal for Traffic and Transport Engineering (IJTTE) 5(1): 74-81.

Andrejić, M.; Kilibarda, M. 2016. A framework for measuring and improving efficiency in distribution channels, International Journal for Traffic and Transport Engineering (IJTTE) 6(2): 137-148.

Bag, S.; Wood, L.C.; Mangla, S.K.; Luthra, S. 2020. Procurement 4.0 and its implications on business process performance in a circular economy, Resources, Conservation \& Recycling 152: 1-14.

Bottani, E.; Centobelli, P.; Murino, T.; Shekarian, E. 2018. A QFD-ANP Method for Supplier Selection with Benefits, Opportunities, Costs and Risks Considerations, International Journal of Information Technology \& Decision Making 17(3): 911-939.

Bressolles, G.; Lang, G. 2019. KPIs for performance measurement of e-fulfillment systems in multi-channel retailing, International Journal of Retail \& Distribution Management 48(1): 35-52.

Chan, F.T.S.; Qi, H.J. 2003. Feasibility of performance measurement system for supply chain: a process-based approach and measures, Integrated Manufacturing Systems 14(3): 179-190.

Krauth, E.; Moonen, H.; Popova, V.; Schut, M. 2005. Performance Indicators in logistics service provision and warehouse management - a literature review and framework. Available from Internet: <https://www. cs.vu.nl/ schut/pubs/Krauth/2005a.pdf>.
Mardani, A.; Nilashi, M.; Zakuan, N.; Loganathan, N.; Soheilirad, S.; Zameri Mat Saman, M.; Ibrahim, O. 2017. A systematic review and meta-Analysis of SWARA and WASPAS methods: Theory and applications with recent fuzzy developments, Applied Soft Computing 57: 265-292.

Radović, D.; Stević, Ž. 2018. Evaluation and selection of KPI in transport using SWARA method, Transport \& Logistics: the International Journal 18(44): 60-68.

Saad, S.; Kunhu, N.; Mohamed, A. 2016. A fuzzy-AHP multi-criteria decision making model for procurement process, International Journal of Logistics Systes and Management 23(1): 1-24.

Shukla, S.; Mishra, P.; Jain, R.; Yadav, H. 2016. An integrated decision making approach for ERP system selection using SWARA and PROMETHEE method, International Journal of Intelligent Enterprise 3: 120-147.

Soni, G.; Kodali, R. 2010. Internal benchmarking for assessment of supply chain performance, Benchmarking: An International Journal 17(1): 44-76.

Stanujkic, D.; Karabasevic, D.; Zavadskas, E.K. 2015. A framework for the Selection of a packaging design based on the SWARA method, Engineering Economics 26: 181-187.

USAID. 2013. Procurement Performance Indicators Guide: Using Procurement Performance Indicators to Strengthen the Procurement Process for Public Health Commodities. Report, Arlington.

Yazdani, M.; Hashemkhani Zolfani, S.; Zavadskas, E.K. 2016. New integration of MCDM methods and QFD in the selection of green suppliers, Journal of Business Economics and Management 17(6): 1097-1113.

Yu, K.; Cadeaux, J.; Song, H. 2012. Alternative forms of fit in distribution flexibility strategies, International Journal of Operations \& Production Management 32(10): $1199-1227$. 